\title{
Color perception is impaired in baseball batters while performing an interceptive action
}

\author{
Manami Sasada $^{1} \cdot$ Hiroki Nakamoto $^{2} \cdot$ Sachi Ikudome $^{2} \cdot$ Satoshi Unenaka $^{3}$. \\ Shiro Mori ${ }^{2}$
}

Published online: 22 April 2015

(C) The Psychonomic Society, Inc. 2015

\begin{abstract}
In order to test the theoretical idea that experts rely more on the dorsal stream than the ventral stream during interceptive action for the interception of a moving target, the present study investigates the perception of color (dominant in ventral processing) during interceptive action in fast-ball sports. Twelve college baseball players and 12 non-baseball players performed a coincident-timing task with target color changes (from white to red, blue, or white) at various time points (at 100, 200, or $300 \mathrm{~ms}$ before target arrival). In this task, participants swung a bat and/or pressed a button in response to the target's arrival at a prespecified location. Participants were then asked to state the final color of the target. Baseball players, but not non-baseball players, were significantly less proficient at identifying color changes during the bat-swing condition relative to the button-press condition, irrespective of the time points of color change. These results are consistent with the idea that baseball players rely more on the dorsal stream during bat swinging for the interception of a moving target than do novices.
\end{abstract}

Keywords Dual visual systems · Sport expertise ·

Visuo-motor control

Hiroki Nakamoto

nakamoto@nifs-k.ac.jp

1 Undergraduate School of Physical Education, National Institute of Fitness and Sports in Kanoya, 1 Shiromizu, Kanoya

891-2393, Kagoshima, Japan

2 Faculty of Physical Education, National Institute of Fitness and Sports in Kanoya, 1 Shiromizu, Kanoya, Kagoshima 891-2393, Japan

3 Graduate School of Physical Education, National Institute of Fitness and Sports in Kanoya, 1 Shiromizu, Kanoya 891-2393, Kagoshima, Japan

\section{Color perception is impaired in baseball batters while performing an interceptive action}

The vast majority of research on sport expertise confirms that experts in fast-ball sports are skilled at perceiving and interpreting essential kinematic information from the early part of an opponent's action sequences prior to ball flight, which enables athletes to anticipate outcomes in advance and circumvent severe time constraints produced by the perceptual-motor system (Abernethy \& Russell, 1987; Jones \& Miles, 1978; for review, Williams, Davids, \& Williams, 1999). However, fastball expertise has recently been discussed in terms of a dual visual system (i.e., ventral and dorsal visual streams) model advocated by Milner and Goodale (1995, 2008). This model suggests that functional differences between the two visual pathways are best understood in terms of the output systems that the two streams serve (Milner \& Goodale, 2008). According to this model, the ventral "vision-for-perception" pathway delivers information about object characteristics, while the dorsal "vision-for-action" stream provides visual information relevant to movement control (Milner \& Goodale, 1995; van der Kamp, Rivas, van Doorn, \& Savelsbergh, 2008). Proponents of this model (van der Kamp et al., 2008; Mann et al., 2010a, b) have stated that the majority of research on anticipation thus far has assessed only the ventral pathway because anticipation tasks often require conscious perceptual responses (i.e., perception-action-uncoupled responses, such as verbal or button pressing) rather than the subconscious motor responses required in actual scenarios (i.e., perceptionaction-coupled responses, such as swinging a racket or bat). Therefore, van der Kamp and colleagues (2008) have claimed that the present understanding of visual anticipation is limited and somewhat biased.

Mann and colleagues (2010a) addressed this criticism by comparing anticipation accuracy in cricket batting between 
uncoupled responses that presumably involve ventral processing, and coupled responses that presumably involve dorsal processing. They reported that anticipation accuracy based on the early part of an opponent's action sequences was greater in the coupled condition relative to the uncoupled condition in experts but not novices. Thus, they concluded that anticipation skill in experts might rely on the dorsal stream when they receive and interpret the anticipatory kinematic information. On the other hand, van der Kamp and colleagues (2008) proposed that while the ventral visual pathway predominantly contributes to anticipation prior to the availability of ball flight information (i.e., the use of precontact information to guarantee interception), the dorsal visual pathway predominantly contributes to the interceptive action following ball release (i.e., making contact with an approaching ball). Indeed, Farrow and Abernethy (2003) reported that superior anticipation in experts was observed under the coupled response condition only when ball flight information was available. Similarly, Mann et al. (2010a) showed that anticipation accuracy and perceptionaction coupling were more pronounced when ball flight information was available. Although these findings may support both the role of the dorsal stream in fast-ball expertise and the predominant contribution of the dorsal stream following ball release, these studies mainly focused on the dorsal contribution to anticipation based on preball flight information (e.g., opponent's kinematic information). Therefore, the dorsal contribution to expertise in interceptive action following ball release has not been demonstrated.

Mann, Abernethy, and Farrow (2010b, c) examined the contribution of the dorsal stream to the interception of fastmoving targets by using visual blur. This was based on the idea that the dorsal visual pathway relies only on visual input from the magnocellular stream, which rapidly conveys information and is highly sensitive to movement and contrast but relatively insensitive to clarity and color. In contrast, the ventral pathway relies on both magnocellular and parvocellular input (Goodale \& Milner, 1992; Goodale, Milner, Jakobson, \& Carey, 1991; Milner \& Goodale, 1995; Ungerleider \& Mishkin, 1982). The parvocellular pathway is sensitive to color and detail (Milner \& Goodale, 1995). Given these functional characteristics, the authors hypothesized that the disparate visual information supplying the two visual pathways offers a potential means to differentially examine the respective visual input. If the ventral visual pathway controls interceptive performance in experts, then decrements in interceptive performance would be expected under a visual blur condition that decreased visual clarity. In contrast, if interception is controlled by the dorsal visual pathway, then performers would demonstrate some degree of resistance to blur. As expected, Mann et al. (2010c) found that low-level visual blur did not affect interceptive performance. This therefore suggests that expert batters rely more on the dorsal stream during interceptive action relative to novices. Thus, experts in fast-ball sports use the two complementary visual systems before and after key events, such as ball release (van der Kamp et al., 2008).

However, previous studies have not sufficiently examined the detailed time course of the dorsal stream's contribution to interception. Studies have demonstrated the existence of a critical time window for interception by using an occlusion technique (Delucia \& Cochran, 1985; Whiting, Alderson, \& Sanderson, 1973; Whiting, Gill, \& Stephenson, 1970). For instance, Müller and Abernethy (2006) examined the ability of cricket batsmen of different skill levels to perceive information from the prerelease and ball flight. In their study, such information was occluded at defined periods through the use of liquid crystal spectacles. Results suggested a superior capability of skilled players to utilize later (postbounce) ball flight information to guide successful bat-ball interception. These studies suggest that there is a critical time window wherein the contribution of the dorsal stream can be enhanced, especially in the latter phase of interception. van der Kamp and colleagues (2008) suggested that the ventral and the dorsal systems are equally engaged during movement onset, and that the relative contribution of the dorsal stream increases around the time of bat-ball contact.

The present study used a coincident-timing task with target color changes. In this task, the moving target changed color during its motion at various time points. Participants were required to swing a bat (coupled response) and/or press a button (uncoupled response) upon target arrival at the interception point. Following the response, participants were asked to state whether the target's color had changed. Anatomical studies indicate that the output from the primary and secondary visual cortices (V1 and V2) project to MT (middle temporal) involving the dorsal stream, and visual area 4 (V4) involving the ventral stream. While the MT is specialized for the processing of motion and depth, V4 is specialized for the processing of form and possibly color (Bear, Connors, \& Paradiso, 2007). Therefore, if the contribution of the dorsal stream increases as interceptive action advances, then color change recognition performance should gradually decrease more in the coupled condition. By contrast, if interception is dominantly controlled by the ventral stream, then performers will demonstrate comparable color change recognition in the coupled and uncoupled conditions. The present study investigates the perception of color at different time points during interceptive action in baseball and non-baseball players.

\section{Method}

\section{Participants}

Twenty-four male college students, aged between 20 and 23 years, participated in this study after giving their informed consent. All participants had normal or corrected-to-normal 
vision and were not color-blind. Twelve participants were college baseball players $(M=13.2 \pm 1.45$ years of experience in baseball) and members of the official college baseball team. The baseball players generally spent $20 \mathrm{~h} /$ week in baseball training, which included batting and fielding, and regularly participated in matches. The other 12 participants (nonbaseball group) were college students who played baseball but had not received any baseball-specific training. Participants were informed of the experimental procedures in advance and consented to take part in this experiment. This study was approved by the ethics committee of the National Institute of Fitness and Sports in Kanoya and was conducted in accordance with the Declaration of Helsinki.

\section{Experimental task and apparatus}

Participants performed a coincident-timing task with target color changes during target motion. A virtual moving target ( $23.5 \mathrm{~cm}$ diameter) was projected on a wall in a gymnastic hall by a projector (XJ-S37; CASIO computer Co. Ltd, Tokyo, Japan) that was connected to a computer that generated the linear motion of the projected objects (see Fig. 1). The target moved from the start to the interception point (a distance of $9 \mathrm{~m})$ on the wall at a constant velocity $(22.5 \mathrm{~m} / \mathrm{s})$ after the presentation of a 3-s warning stimulus. The total time of target presentation was $400 \mathrm{~ms}$, an interval similar to that encountered by a baseball batter during authentic batting (Frohlich, 1984; Selin, 1959; Williams \& Underwood, 1986). In order to prevent variations in batting movement and strategy, which could have served as perceptual confounds during the task, warning time and target velocity were held constant in the present study. In some trials, the target's color suddenly changed from white to red or blue at 100,200 , or $300 \mathrm{~ms}$ before it reached the interception point. In other trials, the target remained the same color from the start to the target arrival point. Participants were required to swing a standard wooden baseball bat (coupled response) and/or press a button (uncoupled response) at the moment of the target's arrival at the interception point. In the coupled response condition, participants were required to swing the bat naturally to mimic an actual batting situation. In the uncoupled response condition, the participants held a manual switch $(10 \mathrm{~cm}$ high, $3 \mathrm{~cm}$ wide, and $2 \mathrm{~cm}$ deep) in the palm of their hand and were asked to push a button with their thumb. Ready posture was the same in both response conditions. After they responded, participants were asked to state whether the target's color had changed. If participants indicated a change in target color, they were subsequently asked to state the color to which it had changed.

In order to estimate coincidence-timing performance during the task, a timer that recorded the duration between start and stop signals was used (AO-MC1 model; Applied Office Co. Ltd, Tokyo, Japan). The start signal was outputted at the start of target movement by the target-generating computer. The stop signal was generated by an infrared sensor that detected the passing of the bat at the arrival point (coupled response task) and/or by the pressing of the button by the

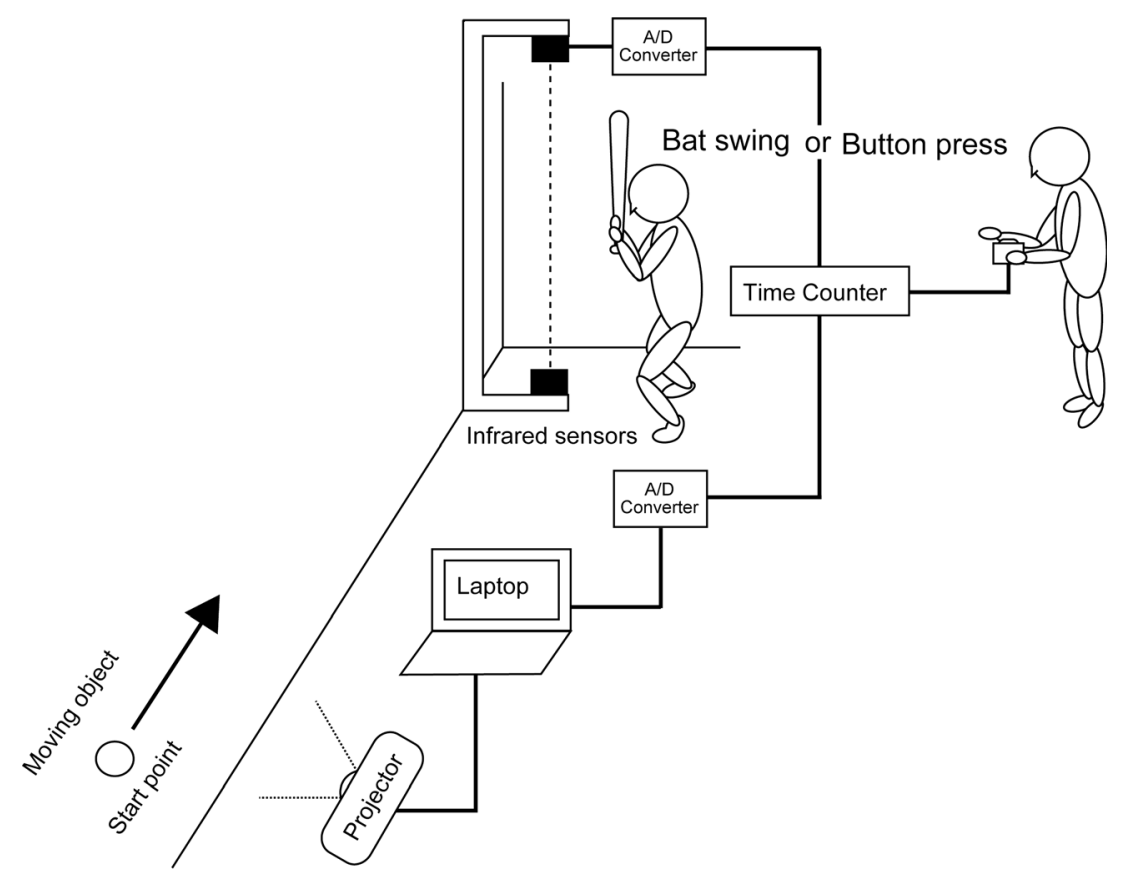

Fig. 1 Schematic representation of the experimental set-up. After a 3-s warning stimulus, the target moved toward the participants at a constant velocity; in some trials, the target's color suddenly changed at 100, 200, or $300 \mathrm{~ms}$ after the target left the starting point. The participants first stood

beside a defined interception point and were asked to swing a bat and/or push a button upon the target's arrival at the interception point. After the response, participants were asked to state whether the target's color had changed. 
participant (uncoupled response task). These stop signal devices were placed at the target arrival point (see Fig. 1). The frame rate of the display projector was $30 \mathrm{~Hz}$, meaning that participants had a 33-ms window during which their response would be correct (i.e., from $367 \mathrm{~ms}$ to $400 \mathrm{~ms}$, after target presentation). Therefore, in the present study, the middle of this time window was designated as the correct time (i.e., $383 \mathrm{~ms}$ ) for target interception. Temporal errors were calculated by subtracting the duration between start and stop signals from the correct time duration.

\section{Experimental procedure}

The batters first stood beside a standard home plate that was placed on the floor, just under the target arrival point. Participants familiarized themselves with the experimental procedure under all conditions, including the changed and unchanged color conditions for each response type. In the subsequent experimental task, the participants performed 40 coincident-timing responses in each of the two response conditions. In each response condition, there were 10 presentations of the four types of stimulus conditions (unchanged, and color change at 100, 200, $300 \mathrm{~ms}$ before target arrival). Color probability (i.e., blue or red) in the color-change conditions was $50 \%$. Stimulus presentation was randomized to offset the effects of environment and subjective expectancy. After responding, participants were asked to judge the color change during ball flight. The interval between trials was set at $10 \mathrm{~s}$, and the block interval was approximately 3-5 min. One concern was that the participants might use different visual search strategies for the moving target depending on the response type (e.g., Land \& Furneaux, 1997), and visual search strategy might have affected performance in color judging. Participants therefore were asked to maintain the same visual search strategy during the task, irrespective of response type.

\section{Behavioral measures and statistics}

We analyzed the absolute temporal error (AE) as an index of accuracy in timing performance. To calculate the temporal error, we measured the time difference between target arrival (i.e., $387 \mathrm{~ms}$ after target presentation) and response (i.e., duration from target presentation to button pressing or bat swinging) at the impact point sampled at $1000 \mathrm{~Hz}$. The percentage of correct responses (CR) with regard to target color was also calculated. The $\mathrm{CR}$ was defined as the percentage of trials in which the participant correctly identified the final color of the ball (white, red, or blue). The mean AE and CR were analyzed using a mixed measures three-way 2 (group: baseball and nonbaseball) $\times 2$ (response type: button press and swing) $\times 4$ (time of change: 100, 200, $300 \mathrm{~ms}$, and no change) ANOVA. The partial effect size $\left(\eta p^{2}\right)$ is reported, and post hoc mean comparisons were performed using the Bonferroni test.

\section{Results}

\section{Temporal error differences in each task condition}

Figure 2 shows the temporal errors of each condition for each group. The three-way ANOVA for AE showed a significant group $\times$ response interaction, $F(1,22)=4.38, p<.05, \eta p^{2}=$ .17. AE was significantly smaller in the baseball group relative to the control group in the coupled condition $(p=.08)$, but not the uncoupled condition. There were no second-order interactions, $F(3,66)=0.46, p=.71, \eta p^{2}=.02$, or first-order interactions for group $\times$ time, $F(3,66)=0.29, p=.83, \eta p^{2}=.01$, or response $\times$ time, $F(3,66)=0.46, p=.71, \eta p^{2}=.02$. There were no main effects of group, $F(1,22)=0.21, p=.65, \eta p^{2}=$ .01 , response, $F(1,22)=0.29, p=.87, \eta p^{2}=.01$, or time, $F(3$, 66) $=1.00, p=.40, \eta p^{2}=.04$.

\section{Detection of color change in each task condition}

Figure 3 shows the $\mathrm{CR}$ in each condition for each group. In the no-change condition, all non-baseball and six baseball players had CRs of $100 \%$. Some of the assumptions for an ANOVA are violated in these data, at least for the "non-baseball players-no change" condition, where performance was perfect (no variability). Therefore, the CRs were analyzed excluding the no change condition in both groups. The threeway ANOVA for CR showed a main effect of time, $F(2,44)=$ $59.41, p<.01, \eta p^{2}=.73$. This main effect indicated that the $\mathrm{CR}$ at $100 \mathrm{~ms}$ in the color-change condition was lower than that of other two time conditions $(p<.01)$. The CR at $200 \mathrm{~ms}$ was also lower than that of the $300 \mathrm{~ms}$ condition $(p<.01)$. Moreover, there was a significant group $\times$ response interaction, $F(1,22)=6.02 p<.05, \eta p^{2}=.22$. The $\mathrm{CR}$ was lower in the coupled response than in the uncoupled response in baseball players $(p<.05)$, but not non-baseball players. There were no main effects of response, $F(1,22)=1.57, p=.22, \eta p^{2}=.07$, or group, $F(1,22)=1.03, p=.32, \eta p^{2}=.05$. There were also no first-order interactions for group $\times$ time, $F(2,44)=0.33, p$ $=.97, \eta p^{2}=.01$, or for response $\times$ time, $F(2,44)=0.13, p=$ $.88, \eta p^{2}=.06$. No second-order interactions were found, $F(2$, 44) $=1.50, p=.24, \eta p^{2}=.06$.

\section{Discussion}

The present study investigated the perception of color at various time points during interceptive action in baseball and non-baseball players during fast-ball sports. The underlying hypothesis for this experiment was that if the contribution of the dorsal stream gradually increases as interceptive action approaches, then color-change recognition proficiency should gradually decrease more in the coupled condition. Furthermore, if baseball players rely more on the dorsal 

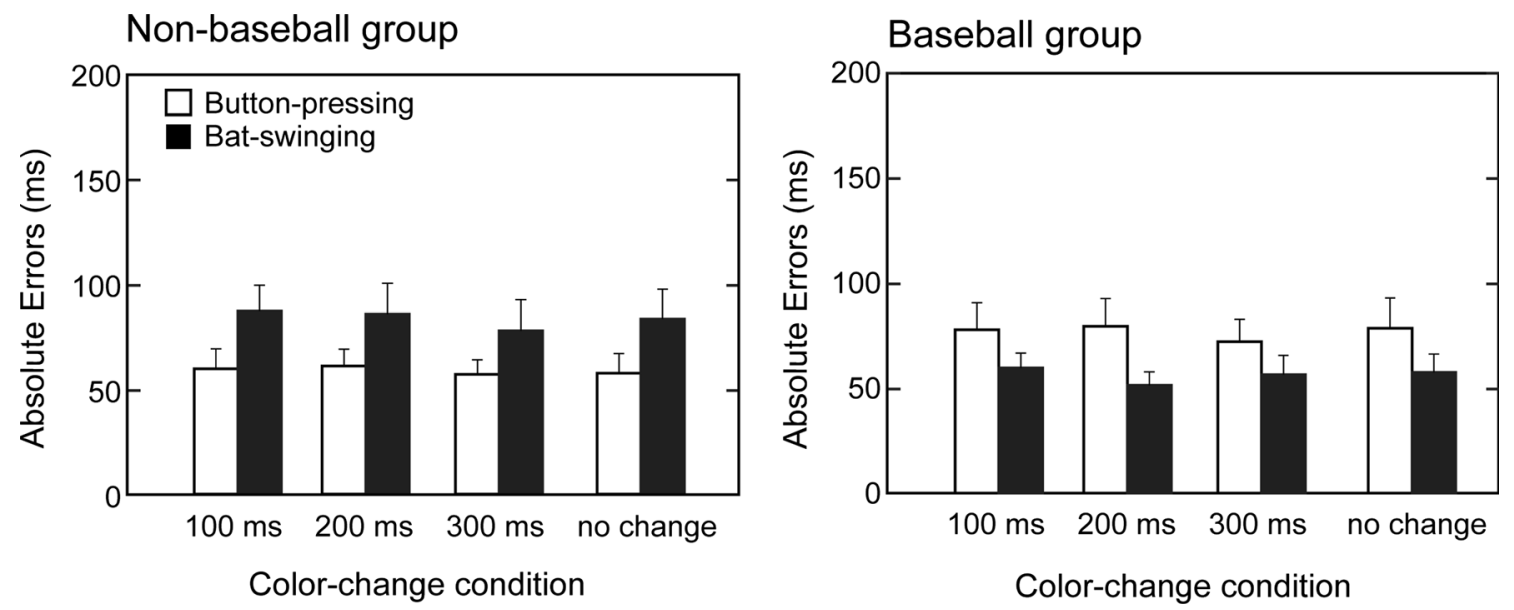

Fig. 2 Mean absolute errors in the baseball and non-baseball groups in each color-change condition. Standard error is indicated by vertical bars.

stream than do non-baseball players, only baseball players should fail to recognize target color change in the coupled condition.

In the present study, the baseball players showed superior timing performance compared with non-baseball players only in a situation quite specific to their expertise (i.e., coupled condition; see Fig. 2). This result suggests that hitting a video projection of a ball with a bat is a good representation of hitting a real one. In a study of sports expertise, Williams and Ericsson (2005) stated that a representative task that could capture expert performance and consequently reflect expertnovice differences is critical to identifying the underlying mechanism of expert performance. Therefore, the present result confirms that the present task is useful for identifying the underlying mechanism of interceptive action.

The main results of this study were that baseball players exhibited a lower CR in the coupled response condition relative to the uncoupled response condition. However, a reduction in $\mathrm{CR}$ in the uncoupled response condition compared with the coupled response condition was not observed in nonbaseball players. Milner and Goodale (2008) stated that the dorsal stream does not use the high-level perceptual object representations constructed by the ventral stream, but instead relies on current, bottom-up information from the retina to dictate the required movement parameters. In addition, as already stated, V4 in the ventral stream is specialized for processing form and possibly color (Bear et al., 2007). Therefore, the present results suggest that ventral processing was decreased in baseball players during bat swinging, but not in non-baseball players. In other words, this is consistent with the idea that experts rely more on the dorsal stream during bat swinging for the interception of a moving target than do novices (van der Kamp et al., 2008). This finding supports recent behavioral evidence that the dorsal visual stream is critically
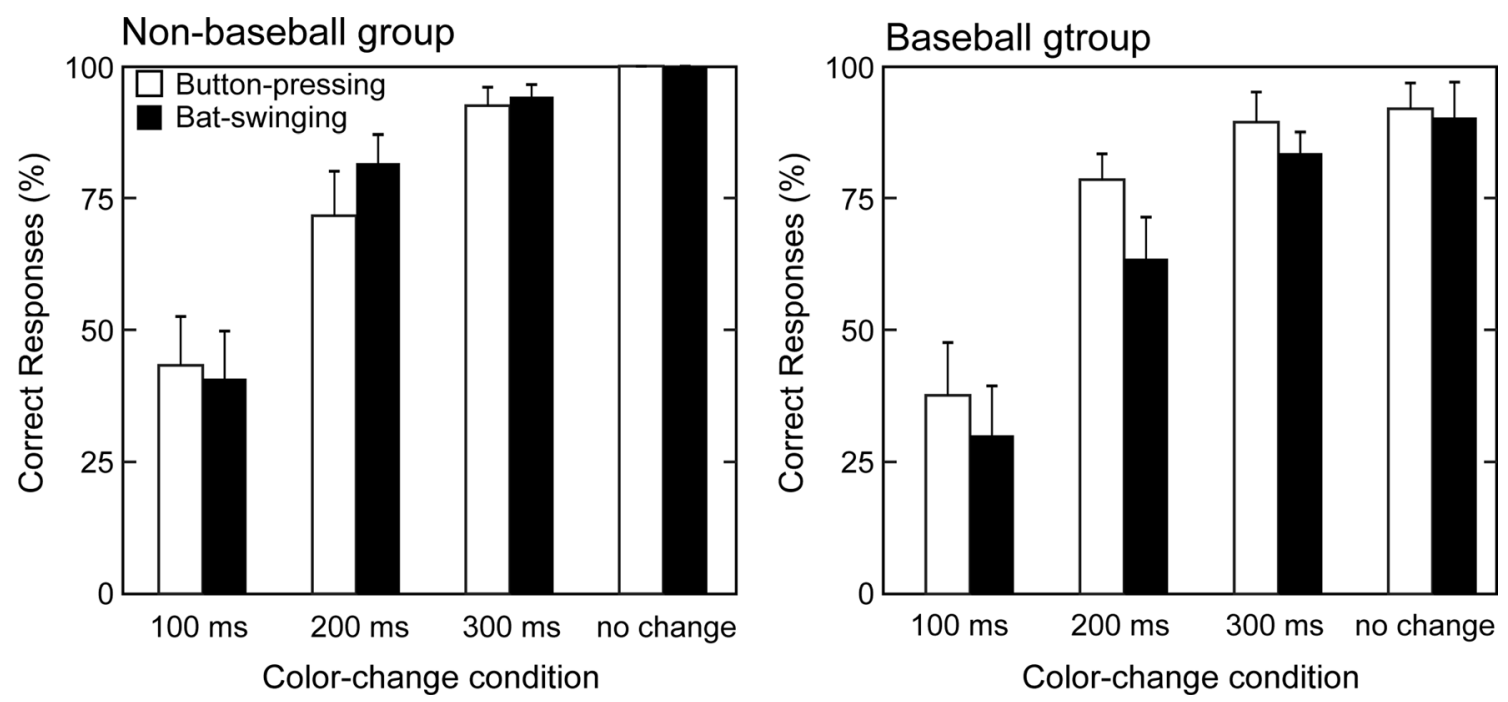

Fig. 3 Mean rate of correct answers in response to target color in both groups for each condition. Standard error is indicated by vertical bars. 
involved in the development of expertise in fast-ball sports (Farrow \& Abernethy, 2003; Mann, Abernethy, \& Farrow, 2010b, c; van der Kamp et al., 2008). van der Kamp et al (2008) suggested that the novice's performance relies heavily on the ventral system until the dorsal system assumes control and movement execution becomes highly automatized. This is supported by evidence that verbalization during action execution appears to encourage the involvement of the ventral system in movement control (Rossetti, 1998; Rossetti \& Pisella, 2002). Therefore, the non-baseball players in the present study did not exhibit CR differences in the coupled and uncoupled conditions.

Conversely, another possible explanation for the CR reduction in baseball players is a difference in visual search strategy for moving objects between novices and experts. Hubbard and Seng (1954) reported that the line of gaze in baseball batters was uncoupled from the ball at $240 \mathrm{~ms}$ prior to bat-ball contact (for similar findings in other sports, see Croft, Button, \& Dicks, 2010; Ripoll \& Fleurance, 1988; Singer et al., 1998). In order to circumvent this limitation, fast-ball experts often exhibit a large anticipatory saccade for ball-tracking until the point of bat-ball contact (Bahill \& LaRitz, 1984; Land \& McLeod, 2000; Ripoll \& Fleurance, 1988; Singer et al., 1998). It is known that visual information is suppressed and/or degraded during saccadic eye movement (Campbell \& Wurtz, 1978; Volkmann, Schick, \& Riggs, 1968). This evidence suggests that the observed CR reduction in baseball players resulted from the anticipatory saccade. If this is the case, an improvement in color perception should be observed in experts at the point of bat-ball contact; however, the present findings did not indicate such an improvement. In addition, Land and McLeod (2000) found that a saccade occurred after the pursuit eye movement during the early phase of ball flight (see also Croft et al., 2010; Mann, Spratford, \& Abernethy, 2013). In the present study, the CR reduction in baseball players had already occurred by early-phase ball flight. Therefore, it is unlikely that the observed reduction in experts' $\mathrm{CR}$ in the coupled condition was due to a difference in visual search strategy such as anticipatory saccade.

It was stated several times that anticipatory saccades are necessary because a ball moves too quickly to be tracked by the eyes. On the other hand, Mann et al. (2013) recently suggested that this is not necessarily the case; rather, head tracking means that the ball could be foveated throughout the majority of ball flight, but instead saccades are still produced and probably serve some other function. In their study, as the ball approached the bat, the gaze of the skilled participants was ahead of the ball. Therefore, the batter relies, at least in part, on peripheral vision to process ball-flight information by using anticipatory saccades. Color vision in the peripheral field is believed to be substantially less developed than color vision in the central field. Given this, the CR reduction in baseball players implies that they perceived the ball-flight information through their peripheral field, which is consistent with Mann's results. As already stated, the dorsal visual pathway relies only on visual input from the magnocellular stream (e.g., Boussaoud, Desimone, \& Ungerleider, 1991; Livingstone \& Hubel, 1987; Shipp \& Zeki, 1985). Wright and Ikeda (1974) reported that the magnocellular ganglion cells were more prevalent in the periphery of the retina (Schiller \& Malpeli, 1978). These findings suggest that ball-flight information conveyed from the peripheral field may enhance dorsal visual processing in baseball players.

Furthermore, CRs in both groups decreased when the color change occurred at a late phase during the interceptive action. This result suggests that the contribution of the ventral stream decreases (and conversely, contribution of the dorsal stream increases) during the latter phase of interception, as modeled by van der Kamp and colleagues (2008). Milner and Goodale (2008) stated that the ventral stream mediates conscious perception and action selection prior to movement onset, and the dorsal stream mediates the detailed specification and online control of the movement after movement onset. The present study supports this notion. On the other hand, a late-phase reduction in CRs occurred in both response conditions. Therefore, this finding may have resulted from factors other than an increased dorsal stream contribution during the latter phase of interception. One alternative factor may have been the limitation inherent in the visual tracking of task stimuli. Previous studies in fast-ball sports have demonstrated that batters cannot track the ball flight path until bat-ball contact during the batting act (Bahill \& LaRitz, 1984; Hubbard \& Seng, 1954; Land \& McLeod, 2000; Mann et al., 2013). Thus, the participants in the present study may have been less proficient at detecting color change during late-phase interception because they were unable to track the target until the end of ball flight. Yet, the reduction in CRs in baseball players occurred during the early phase, suggesting that they could track the ball and that the late-phase reduction in CRs was not solely due to this limitation in visual tracking.

In conclusion, this study examined the perception of color over the time course of interception and investigated expertnovice differences in reliance on the visual streams. To this end, the present study used a coincident-timing task with target color changes. Results showed a significant reduction in color-change detection in the coupled response relative to the uncoupled response in baseball players. These results are consistent with the idea that experts rely more on the dorsal stream during bat swinging for the interception of a moving target than do novices.

\section{Limitations}

In interpreting the results, we assumed that if the dorsal system were dominant when hitting a target, then the ventral system's capacity for color change detection would decrease. However, 
in order to confirm this assumption, it is necessary to posit that the two visual streams have a completely complementary relationship. Therefore, the present results may only indicate that the baseball players decrease ventral processing during bat swinging. Moreover, the present study could not entirely exclude the possible involvement of visual search strategy in the observed reduction in CRs. In other words, the present study does not unequivocally confirm that the contribution of the dorsal stream increases and/or that of the ventral stream decreases with the progression of interceptive action. Therefore, further investigations are needed to confirm the present interpretation by recording gaze behavior during the task. Finally, although the present task was, to some extent, domain-specific to baseball, the target, distance from the interception point, velocity, and interception response are low in representative task design for a baseball test. For instance, the use of a virtual ball may preferentially activate the dorsal stream. Goodale, Jakobson, and Keillor (1994) argue that pantomime action may actually involve ventral rather than dorsal stream contributions (see also Westwood, Chapman, \& Roy, 2000). Although the results showed some degree of an expertise effect, the extent to which this effect was influenced by the lack of specificity within the experimental design is unclear. Therefore, further experiments (e.g., in situ test) are needed to fully understand the effects of expertise on visual stream contributions during interceptive action.

Author Note Hiroki Nakamoto, Faculty of Physical Education. National Institute of Fitness and Sports in Kanoya.

\section{References}

Abernethy, B., \& Russell, D. G. (1987). The relationship between expertise and visual search strategy in a racquet sport. Human Movement Science, 6, 283-319.

Bahill, A. T., \& LaRitz, T. (1984). Why can't batters keep their eyes on the ball? American Scientist, 72, 249-253.

Bear, M., Connors, B., \& Paradiso, M. (2007). Neuroscience: Exploring the brain (3rd ed.). Philadelphia, PA: Lippincott Williams \& Wilkins.

Boussaoud, D., Desimone, R., \& Ungerleider, L. G. (1991). Visual topography of area TEO in the macaque. The Journal of Comparative Neurology, 306, 554-575.

Campbell, F. W., \& Wurtz, R. H. (1978). Saccadic omissions: Why we do not see a grey out during a saccadic eye movement. Vision Research 18, 1297-1303.

Croft, J. L., Button, C., \& Dicks, M. (2010). Visual strategies of sub-elite cricket batsmen in response to different ball velocities. Human Movement Science, 29, 751-763.

Delucia, P. R., \& Cochran, E. L. (1985). Perceptual information for batting can be extracted throughout a ball's trajectory. Perceptual \& Motor Skills, 61, 143-150.

Farrow, D., \& Abernethy, B. (2003). Do expertise and the degree of perception-action coupling affect natural anticipatory performance? Perception, 32, 1127-1139.
Frohlich, C. (1984). Aerodynamics drag crisis and its possible effect on the flight of baseballs. American Journal of Physics, 52, 325-334.

Goodale, M. A., Jakobson, L. S., \& Keillor, J. M. (1994). Differences in the visual control of pantomimed and natural grasping movements. Neuropsychologia, 32, 1159-1178.

Goodale, M. A., \& Milner, A. D. (1992). Separate visual pathways for perception and action. Trends in Neuroscience, 15, 20-25.

Goodale, M. A., Milner, A. D., Jakobson, L. S., \& Carey, D. P. (1991). A neurological dissociation between perceiving objects and grasping them. Nature, 349, 154-156.

Hubbard, A. W., \& Seng, C. N. (1954). Visual movements of batters. Research Quarterly, 25, 42-57.

Jones, C. M., \& Miles, T. R. (1978). Use of advance cues in predicting the flight of a lawn tennis ball. Journal of Human Movement Studies, 4, 231-235.

Land, M. F., \& Furneaux, S. (1997). The knowledge base of the oculomotor system. Philosophical Transactions of the Royal Society of London. Series B: Biological Sciences, 352, 1231-1239.

Land, M. F., \& McLeod, P. (2000). From eye movements to actions: How batsmen hit the ball. Nature Neuroscience, 3, 1340-1345.

Livingstone, M. S., \& Hubel, D. H. (1987). Psychophysical evidence for separate channels for the perception of form, color, movement, and depth. Journal of Neuroscience, 7, 3416-3468.

Mann, D. L., Abernethy, B., \& Farrow, D. (2010a). Action specificity increases anticipatory performance and the expert advantage in natural interceptive tasks. Acta Psychologica, 135, 17-23.

Mann, D. L., Abernethy, B., \& Farrow, D. (2010b). Visual information underpinning skilled anticipation: The effect of blur on a coupled and uncoupled in situ anticipatory response. Attention, Perception, \& Psychophysics, 72, 1317-1326.

Mann, D. L., Abernethy, B., \& Farrow, D. (2010c). The resilience of natural interceptive actions to refractive blur. Human Movement Science, 29, 386-400.

Mann, D. L., Spratford, W., \& Abernethy, B. (2013). The head tracks and gaze predicts: How the world's best batters hit a ball. PLOS One, 8 , e58289.

Milner, A. D., \& Goodale, M. A. (1995). The visual brain in action. Oxford, England: Oxford University Press.

Milner, A. D., \& Goodale, M. A. (2008). Two visual systems re-viewed. Neuropsychologia, 46, 774-785.

Müller, S., \& Abernethy, B. (2006). Batting with occluded vision: An in situ examination of the information pick-up and interceptive skills of high- and low-skilled cricket batsmen. Journal of Science and Medicine in Sport, 9, 446-458.

Ripoll, H., \& Fleurance, P. (1988). What does keeping one's eye on the ball mean? Ergonomics, 31, 1647-1654.

Rossetti, Y. (1998). Implicit short-lived motor representations of space in brain damaged and healthy subjects. Consciousness and Cognition, 7, 520-558.

Rossetti, Y., \& Pisella, L. (2002). Several 'vision for action' systems: A guide to dissociating and integrating dorsal and ventral functions. In W. Prinz \& B. Hommel (Eds.), Attention and performance XIX: Common mechanisms in perception and action(pp (pp. 62-119). Oxford, England: Oxford University Press.

Schiller, P. H., \& Malpeli, J. G. (1978). Functional specificity of lateral geniculate nucleus laminae of the rhesus monkey. Journal of Neurophysiology, 41, 788-797.

Selin, C. (1959). An analysis of the aerodynamics of pitched balls. Research Quarterly for Exercise and Sport, 30, 232-240.

Shipp, S., \& Zeki, S. (1985). Segregation of pathways leading from area V2 to areas V4 and V5 of macaque monkey visual cortex. Nature, $315,322-325$.

Singer, R. N., Williams, A. M., Frehlich, S. G., Janelle, C. M., Radlo, S. J., Barba, D. A., \& Bouchard L. J. (1998) New frontiers in visual search: 
An exploratory study in live tennis situations. Research Quarterly for Exercise and Sport, 69, 290-296.

Ungerleider, L. G., \& Mishkin, M. (1982). Two cortical visual systems. In D. J. Ingle, M. A. Goodale, \& R. J. W. Mansfield (Eds.), Analysis of visual behavior (pp. 549-586). Cambridge, MA: MIT Press.

van der Kamp, J., Rivas, F., van Doorn, H., \& Savelsbergh, G. (2008). Ventral and dorsal system contributions to visual anticipation in fast ball sports. International Journal of Sport Psychology, 39, 100-130.

Volkmann, F. C., Schick, A. M., \& Riggs, L. A. (1968). Time course of visual inhibition during voluntary saccades. Journal of the Optical Society of America, 58, 562-569.

Westwood, D. A., Chapman, C. D., \& Roy, E. A. (2000). Pantomimed actions may be controlled by the ventral visual stream. Experimental Brain Research, 130, 545-548.

Whiting, H. T. A., Alderson, G. J. K., \& Sanderson, F. H. (1973). Critical time intervals for viewing and individual differences in performance of a ball-catching task. International Journal of Sport Psychology, $46,420-424$

Whiting, H. T. A., Gill, E. B., \& Stephenson, J. M. (1970). Critical time intervals for taking in flight information in a ball-catching task. Ergonomics, 13, 265-272.

Williams, A. M., Davids, K., \& Williams, J. G. (1999). Visual perception \& action in sport. London: E. \& F. N. Spon.

Williams, A. M., \& Ericsson, K. A. (2005). Perceptual-cognitive expertise in sport: Some considerations when applying the expert performance approach. Human Movement Science, 24, 283-307.

Williams, T., \& Underwood, J. (1986). The science of hitting. New York, NY: Simon and Schuster.

Wright, M., \& Ikeda, H. (1974). Processing of spatial and temporal information in the visual system. In F. Schmitt \& F. Worden (Eds.), The neurosciences (Third Study Program) (pp. 115-122). Cambridge, MA: MIT Press. 